\title{
Design of Near Perfect Reconstruction Non-Uniform Filter Banks
}

\author{
Fabrizio Argenti \\ Benedetto Brogelli \\ Enrico Del Re
}

\begin{abstract}
Subband coding is widely used to compress speech, audio and video signals. In some applications, for example audio compression, a non-uniform splitting of the spectrum of a digital signal can be preferable to a uniform one. Some techniques that allow to implement a non-uniform width subband decomposition are here discussed, design methods based on the cosine-modulation of more than one prototype are described and some examples of filter bank design are given.
\end{abstract}

\subsection{Introduction}

Splitting the spectrum of a digital signal [1]-[4] can be useful in several applications, for example data compression. Most of the literature in the field of filter banks design is concerned with uniform width subbands. However, in some cases a non-uniform splitting is more suitable, for example in audio coding [5][6], where non-uniform width subbands could match better the critical bands of the human auditory system.

In subband coding applications an analysis filter bank is used to represent a digital signal $x(n)$ through a set of subsequences, each related to a particular frequency interval. Because of the reduced spectrum occupancy, the subband signals can be downsampled (or decimated). Quantization and coding of the subbands samples allow their transmission through the communication channel. At the receiver side, after decoding and inverse quantization, the subband signals are upsampled (or interpolated) and filtered by the synthesis bank, that eliminates the images of the subband spectra produced by the upsampling operation. Summing up all the filtered subbands yields the reconstructed signal $\hat{x}(n)$.

Different types of errors can affect the reconstructed signal: if a rational transfer function $T(z)$ from $x(n)$ to $\hat{x}(n)$ exists, then aliasing error is absent (in general this is not true, since the upsampling and the downsampling operators are linear but time-varying); in the absence of aliasing, the transfer function $T(z)$ should be an allpass and linear phase function, that is a delay: if these properties are 
not satisfied, magnitude and/or phase distortion can affect $\hat{x}(n)$.

In uniform filter banks the downsampling/upsampling factors are all equal to an integer $M$, so that uniform width subbands, covering $1 / M$ of the global spectrum, are produced. If a non-uniform splitting is to be designed, then the scheme presented in Fig. 12.1, which shows a filter bank with rational sampling factors, can be used. At the analysis side of the $m$-th branch a fraction of the global spectrum equal to $R_{m} / M_{m}$ is extracted. If $R_{m}=1, m=0, \ldots, \mathrm{M}-1$, then integer decimation factors are considered.

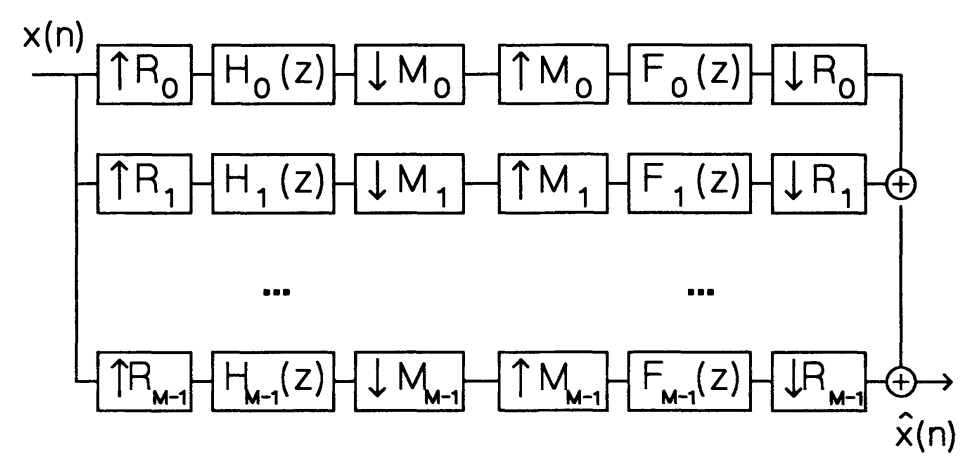

FIGURE 12.1. Non-uniform filter banks with rational decimation factors

The theory of uniform filter banks is well established and several design methods are reported in the literature. A lower number of works appears in the field of non-uniform filter bank design: this problem has been addressed, for example, in [7]-[14].

In [8] the problem of perfect aliasing cancellation in non-uniform filter banks with integer decimation factors is considered. The notion of compatible sets is introduced: the choice of a compatible set as integer decimation factors represents a necessary condition for the cancellation of aliasing when non-ideal filters are taken into account.

In [9] the design of non-uniform filter banks is performed in the time-domain. The reconstructed signal is expressed through the impulse responses of the analysis/synthesis filters. The filters are designed through numerical optimization that minimizes, in some sense, the reconstruction error.

In [10] the theory of Perfect Reconstruction (PR) non-uniform filter banks is described. Two transforms that allow to represent such banks through a filter bank with uniform sampling factors are described and examples of the application of these transforms are given.

In [11] cosine modulation of a low-pass prototype is used to design an uniform 